Gynäkologe 2009 • 42:401

DOI 10.1007/s00129-009-2366-2

Online publiziert: 27. April 2009

(c) Springer Medizin Verlag 2009

R. Kreienberg ${ }^{1}$ - W. Distler ${ }^{2}$. W. Jonat ${ }^{3}$. G. Sauer ${ }^{1}$

${ }^{1}$ Universitätsfrauenklinik und Poliklinik, Ulm

${ }^{2}$ Universitätsfrauenklinik und Poliklinik, Dresden

${ }^{3}$ Universitätsfrauenklinik und Poliklinik, Schleswig Holstein, Campus Kiel

\title{
Neue Aspekte der Strahlentherapie in der Gynäkologie
}

Nachdem die Teilbrustbestrahlung (PBR) unter Nutzung der Multikathetertechnik eine besondere Stellung einnimmt, welche weder eindeutig der IORT noch der üblichen adjuvanten Strahlentherapie nach Brusterhalt zuzuordnen ist, wird Dr. Kovács uns einen Überblick darüber geben, welchen Stellenwert eine solche Methode in Zeiten der sich entwickelnden IORT besitzt.

Immer wieder wird bei der adjuvanten Behandlungsplanung von Uterussarkomen darüber diskutiert, ob und in welcher Weise Patientinnen von einer Strahlentherapie profitieren. Dabei ist es wichtig zu unterscheiden, um welche Art von Sarkom - Leiomyo- oder Karzinosarkom - es sich handelt, wie Frau PD Dr. Marnitz u. a. in ihrem Beitrag erläutern.

Auch in der Behandlung des Vulvakarzinoms ringt man um adjuvante Behandlungskonzepte, nicht zuletzt wegen der "Rezidivfreudigkeit“ dieser Tumorentität. Ist aber deswegen in allen Fällen eine adjuvante Strahlentherapie - mit all ihren Nebenwirkungen und sich daraus ergebenden Einschränkungen - für die $\mathrm{Pa}$ tientin indiziert? Frau Professor Hänsgen wird uns dazu einen kritischen Überblick verschaffen. Strahlentherapie nach brusterhaltender Operation scheint sich eine Abkehr von der Radikalität zu vollziehen. Dr. Bottke wird zum derzeitigen Stand der intraoperativen Radiotherapie (IORT) des Mammakarzinoms eine Übersicht geben, unterschiedliche Methoden beschreiben und bewerten sowie zu den Perspektiven einer solchen neuen Applikationsart Stellung beziehen.

\section{จ Für die adjuvante Bestrahlung des Endometriumkarzinoms ist das Stadium pT1bG2 der "Scheidepunkt"}

Nicht weniger Diskussionsbedarf stellt sich bei der adjuvanten Strahlentherapie des Endometriumkarzinoms. So scheint das Stadium pT1bG2 der "Scheidepunkt“ zu sein: Ist hier die adjuvante Strahlentherapie indiziert? Wenn ja, als Brachytherapie oder perkutane Beckenbestrahlung? Oder ist sie schlichtweg eine Übertherapie, assoziiert mit nicht zu tolerierenden Nebenwirkungen? Sollte man stattdessen besser die pelvine und paraaortale Lymphonodektomie durchführen? Zu diesen Fragen wird uns Dr. Varga einen kritischen Überblick über den derzeitigen Stand der Literatur geben.

Abschließend wird uns Dr. Krause über die radiobiologische Rationale in der strahlentherapeutischen Behandlung gynäkologischer Tumore informieren.

Wir hoffen, mit der Themenauswahl Ihr Interesse zu wecken und aktuelle, teils heftig umstrittene Diskussionspunkte adäquat beleuchtet zu haben.

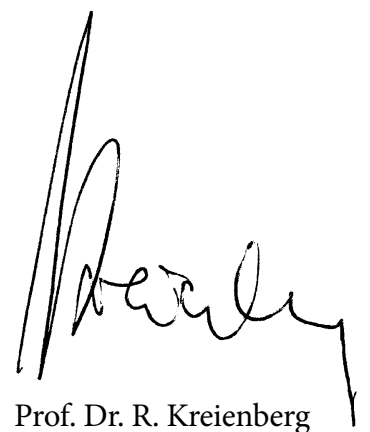

\section{Korrespondenzadresse \\ Prof. Dr. R. Kreienberg \\ Universitätsfrauenklinik und Poliklinik \\ Prittwitzstr. 43, 89075 Ulm \\ Rolf.Kreienberg@uniklinik-ulm.de}

